\title{
A Novel Exact Plate Theory for Bending Vibrations Based on the Partial Differential Operator Theory
}

\author{
Chuanping Zhou ${ }^{1,2,3}$, Maofa Wang ${ }^{1, *}$, Xiao Han ${ }^{3}$, Huanhuan Xue ${ }^{1}$, Jing Ni $^{1}$ and Weihua Zhou ${ }^{4}$ \\ 1 School of Mechanical Engineering, Hangzhou Dianzi University, Hangzhou 310018, China; \\ zhoucp@hdu.edu.cn (C.Z.); weiyangchaohu@163.com (H.X.); nj2000@hdu.edu.cn (J.N.) \\ 2 School of Mechatronics Engineering, University of Electronic Science and Technology of China, \\ Chengdu 611731, China \\ 3 Hangzhou Changchuan Technology Co., Ltd., Hangzhou 310018, China; hanxiao@hzcctech.net \\ 4 College of Electrical Engineering, Zhejiang University, Hangzhou 310027, China; dididi@zju.edu.cn \\ * Correspondence: wmf@hdu.edu.cn; Tel.: +86-134-2916-8099
}

Citation: Zhou, C.; Wang, M.; Han, X.; Xue, H.; Ni, J.; Zhou, W. A Novel Exact Plate Theory for Bending Vibrations Based on the Partial Differential Operator Theory. Mathematics 2021, 9, 1920. https:// doi.org/10.3390/math9161920

Academic Editor: Francisco Beltran-Carbajal

Received: 24 May 2021

Accepted: 2 August 2021

Published: 12 August 2021

Publisher's Note: MDPI stays neutral with regard to jurisdictional claims in published maps and institutional affiliations.

Copyright: (c) 2021 by the authors. Licensee MDPI, Basel, Switzerland. This article is an open access article distributed under the terms and conditions of the Creative Commons Attribution (CC BY) license (https:// creativecommons.org/licenses/by/ $4.0 /)$.

\begin{abstract}
Thick wall structures are usually applied at a highly reduced frequency. It is crucial to study the refined dynamic modeling of a thick plate, as it is directly related to the dynamic mechanical characteristics of an engineering structure or device, elastic wave scattering and dynamic stress concentration, and motion stability and dynamic control of a distributed parameter system. In this paper, based on the partial differential operator theory, an exact elasto-dynamics theory without assumptions for bending vibrations is presented by using the formal solution proposed by Boussinesq-Galerkin, and its dynamic equations are obtained under appropriate gauge conditions. The exact plate theory is then compared with other theories of plates. Since the derivation of the dynamic equation is conducted without any prior assumption, the proposed dynamic equation of plates is more exact and can be applied to a wider frequency range and greater thickness.
\end{abstract}

Keywords: exact plate theory; thick plate; bending vibration; partial differential operator theory; gauge condition

\section{Introduction}

As typical structures, thick plates with holes are widely used under dynamic loads in aerospace, ocean engineering, civil engineering and mechanical engineering [1-5]. Due to the stress concentration near the load-bearing opening in the finite thickness structure, there will be an intense three-dimensional effect zone. The three-dimensional effect zone is closely related to the relative thickness of the structure, which largely controls the fracture, fatigue and other mechanical properties of the structure [6,7]. There is a large error in calculating the dynamic problem of the plate with actual thickness based on the classical thin plate theory. With the development of modern science and technology, engineering structure designs tend to be light, and the way to achieve this is to use advanced materials and improve the structure design theory. However, because the three-dimensional problem has not been well solved, it will encounter great mathematical difficulties in solving the three-dimensional problem.

The classical plate theory (CPT) is the basic theory in the hierarchy of plate theories [6]. Since it was formulated systematically in the 19th century, CPT has been widely applied for buckling, bending and vibration analyses of plates. However, it is worth noting that CPT does not consider the effects of shear deformation or rotary inertia [8-10]. Hence, in the analysis of thick plates and also in the case of thin plates vibrating at higher frequencies, the use of CPT would result in considerable errors [11-14]. These limitations of CPT led to the development of first-order and higher-order shear deformation plate theories.

Reissner [11,12] proposed a thick plate theory, including the effects of shear deformation. Reissner's theory involves three coupled governing differential equations in terms of 
three unknowns. In fact, Reissner's theory is based on a stress approach. Mindlin [13] considered both the effects of shear deformation and rotary inertia and proposed a first-order displacement-based theory, which involves three coupled governing differential equations. Mindlin's theory was derived by using the frequency domain method, so its disadvantage is that it cannot predict the constant transverse shear strains and stresses across the plate thickness. Therefore, the theory requires a shear correction factor to match the strain energy calculated by using constant transverse shear strains and stresses. The transverse shear strains and stresses vary parabolically across the plate thickness [14].

The Levinson [15] and Reddy [16,17] plate theories are higher-order shear deformation plate theories based on displacement. Both the theories are based on the same displacement field. However, the governing equations of the Levinson theory are derived from the plate gross equilibrium equations, whereas Reddy used the principle of virtual displacements to derive the governing differential equations. Neither theory requires a shear correction factor. The plate theory proposed by Kant [18] is based on a higher-order displacement model, which causes a secondary change in transverse shear strain and a linear change in transverse normal strain across the plate thickness. This work involves the flexure of thick rectangular isotropic plates. The formulation of the theory involves six variables. Since the higher-order plate theories cannot take the continuity conditions of displacement and shear stresses into consideration, the accuracy of analytical solutions cannot meet the requirements in engineering applications [19].

Nedri [20] presented a novel refined hyperbolic shear deformation theory based on the assumption that the transverse displacements consist of shear and bending components where the bending components do not contribute to shear forces, and likewise, the shear components do not contribute to bending moments. The irrelevancies of the two components make calculations simple, yet it may lead to an erroneous result when the displacements vary sharply across the thickness. Shimpi [21] developed a refined plate theory (RPT) with only two unknown functions available in the paper. RPT produces two fourth-order governing differential equations, which are uncoupled for static problems and are only inertially coupled for dynamic problems. Subsequently, Shimpi [22] presented two new first-order shear deformation plate theories with only two unknown functions to improve RPT. Using the method proposed by Timoshenko and Ashwell, Nicassio [4] presented a novel forecast model to map the surface profiles of bistable laminates and developed an analytical model to provide an interpretation of the bistable shapes in terms of principal and anticlastic curvatures. $\mathrm{Wu}[5]$ gave a revised method to increase the stiffness and natural frequency of bistable composite shells, which can be suitable for spatial, lightweight structural components.

Among various refined plate theories, Carrera [23] presented a unified expression, which provides a program to obtain refined structural models of beams, plates and shells that explain variable kinematics descriptions. Based on Carrera's unified formulation (CUF), these structural models are obtained using the $\mathrm{N}$-order Taylor expansion to expand the unknown displacement variables. Tornabene et al. [24,25] derived a general formulation of 2D higher-order equivalent plate theory. The theoretical framework covers the static and dynamic analysis of shell structures by using a general displacement field based on CUF. In the CUF system, a linear case can describe a classical model, while a higherorder case can describe a three-dimensional structure. Kolahchi [3] investigated bending, buckling and buckling of embedded nano-sandwich plates based on refined zigzag theory (RZT), sinusoidal shear deformation theory (SSDT), first-order shear deformation theory (FSDT) and classical plate theory (CPT), and a differential cubature (DC) method is applied for obtaining the static response, the natural frequencies and the buckling loads of nano-sandwich plates. The numerical investigation shows that RZT is highly accurate in predicting the deflection, frequency and buckling load of nano-sandwich plates without requiring any shear correction factors.

In brief, although the above CPT theories have been optimized and updated, they are theoretically based on the geometric method, and the models are rough since engineering 
assumptions are still used during the derivation, which results in many limitations in the application of thick wall structures, especially in the case of plates vibrating at higher frequencies. In this paper, we propose a novel theory of exact elastic dynamics for bending plates not based on the geometric view but the algebraic view. During the derivation, we apply the general formal solution proposed by Boussinesq-Galerkin and the operator theory of partial differential equations. The exact elasto-dynamics equations for bending plates are obtained by using appropriate gauge conditions, and the exact dynamic theory of thick plates is compared with other plate theories. Since the derivation of the dynamic equation is carried out without any prior assumption, the proposed dynamic equation of plates is more exact and can be applied in a wider frequency range and greater thickness. The exact thick plate theory in this paper makes up for the shortcomings of the classical thin plate theory and other thick plate theories. It can be used not only for structures with large thickness span ratio but also for vibration mechanics problems with great influence of shear deformation and moment of inertia, such as spacecraft attitude dynamics and control, structural motion stability, dynamic stress concentration for thick plates with holes, and calculations for submarine anechoic tile structure design.

\section{Derivation of the Exact Dynamic Theory for the Bending Plate}

First, the derivation process of the exact plate theory for the bending plate is introduced. According to the three-dimensional elasto-dynamics theory, the governing equation of the spatial displacement field is the Navier equation:

$$
\mu \nabla^{2} \mathbf{u}+(\lambda+\mu) \nabla(\nabla \cdot \mathbf{u})=\rho \frac{\partial^{2} \mathbf{u}}{\partial t^{2}}
$$

where $\mu, \lambda$ are the Lame constants, $\nabla=\mathbf{i} \partial / \partial x+\mathbf{j} \partial / \partial y+\mathbf{k} \partial / \partial z, \rho$ is the density.

From Equation (1), based on Boussinesq-Galerkin solution (B-G solution), the solution given as:

$$
\mathbf{u}=2(1-v)\left(\nabla^{2}-\frac{1}{c_{1}^{2}} \frac{\partial^{2}}{\partial t^{2}}\right) \mathbf{G}-\nabla(\nabla \cdot \mathbf{G}),
$$

where $c_{1}, c_{2}$ are longitudinal wave velocity and transverse wave velocity, $v$ is the Poisson ratio, and $\mathbf{G}=\left(G_{1}, G_{2}, G_{3}\right)$ is the Somigliana vector potential function, which satisfies the following relation as:

$$
\prod\left(\nabla^{2}-T_{j}^{2}\right) \mathbf{G}=0
$$

where $T_{j}$ are time differential operators, $T_{j}^{2}=\frac{1}{c_{j}^{2}} \frac{\partial^{2}}{\partial t^{2}},(j=1,2)$,

Using the Taylor series expansion of the exponential operator function, the displacement at any point in the plate can be written as:

$$
\begin{aligned}
& u_{x}(x, y, z)=\exp \left(z \frac{\partial}{\partial z}\right) u_{x}(x, y, 0) \\
& u_{y}(x, y, z)=\exp \left(z \frac{\partial}{\partial z}\right) u_{y}(x, y, 0) \\
& u_{z}(x, y, z)=\exp \left(z \frac{\partial}{\partial z}\right) u_{z}(x, y, 0) .
\end{aligned}
$$

The fluctuation of plate bending is a case of antisymmetric motion, and Equation (4) can be written as:

$$
\begin{aligned}
& u_{x}(x, y, z)=\sinh \left(z \frac{\partial}{\partial z}\right) u_{x}(x, y, 0), \\
& u_{y}(x, y, z)=\sinh \left(z \frac{\partial}{\partial z}\right) u_{y}(x, y, 0),
\end{aligned}
$$




$$
u_{z}(x, y, z)=\cosh \left(z \frac{\partial}{\partial z}\right) u_{z}(x, y, 0),
$$

where $\sinh (\cdot)$ is hyperbolic sine function, and $\cosh (\cdot)$ is a hyperbolic cosine function.

The B-G solution can be written as:

$$
\begin{aligned}
& G_{j}(x, y, z)=\sinh \left(z \frac{\partial}{\partial z}\right) G_{j}(x, y, 0)=\cosh \left(z \frac{\partial}{\partial z}\right) \sum_{i=1}^{2} G_{j}^{i}(x, y, 0), \\
& G_{3}(x, y, z)=\cosh \left(z \frac{\partial}{\partial z}\right) G_{3}(x, y, 0)=\cosh \left(z \frac{\partial}{\partial z}\right) \sum_{i=1}^{2} G_{3}^{i}(x, y, 0),
\end{aligned}
$$

The Somigliana vector potential function $\mathrm{G}$ can be decompose into two vector potential function as $\mathbf{G}=\mathbf{G}^{1}+\mathbf{G}^{2}$, and $\left(\nabla_{j}^{2}+\frac{\partial^{2}}{\partial z^{2}}\right) \mathbf{G}^{j}=0$, here $\nabla_{j}^{2}=\nabla^{2}-T_{j}^{2},(j=1,2)$ is the Lorentz operator. The trigonometric function operator can be written as:

$$
\begin{gathered}
\frac{\sin \left(z \nabla_{j}\right)}{\nabla_{j}}=\sum_{n=0}^{\infty}(-1)^{n} \frac{1}{(2 n+1) !} z^{2 n+1} \nabla_{j}^{2 n}, \\
\cos \left(z \nabla_{j}\right)=\sum_{n=0}^{\infty}(-1)^{n} \frac{1}{(2 n) !} z^{2 n} \nabla_{j}^{2 n},
\end{gathered}
$$

here $j=1,2$.

We can also obtain the following relation as:

$$
\nabla_{0} \mathbf{G}=\frac{\partial G_{1}}{\partial x}+\frac{\partial G_{2}}{\partial y}+\frac{\partial G_{3}}{\partial z}=\sum_{j=1}^{2} \frac{\sin \left(z \nabla_{j}\right)}{\nabla_{j}}\left(\frac{\partial g_{1}^{j}}{\partial x}+\frac{\partial g_{2}^{j}}{\partial y}-\nabla_{j}^{2} g_{3}^{j}\right) .
$$

For the sake of avoiding the non-uniqueness of unknown functions, two gauge conditions are adopted as follows:

$$
\frac{\partial g_{1}^{j}}{\partial x}+\frac{\partial g_{2}^{j}}{\partial y}=0,(j=1,2)
$$

Equation (8) can be written as:

$$
\begin{aligned}
\nabla_{0}\left(\nabla_{0} \cdot \mathbf{G}\right) & =-\sum_{j=1}^{2}\left[\frac{\sin \left(z \nabla_{j}\right)}{\nabla_{j}} \nabla_{j}^{2} \frac{\partial}{\partial x} g_{3}^{j}\right] \mathbf{i}-\sum_{j=1}^{2}\left[\frac{\sin \left(z \nabla_{j}\right)}{\nabla_{j}} \nabla_{j}^{2} \frac{\partial}{\partial y} g_{3}^{j}\right] \mathbf{j} \\
& -\sum_{j=1}^{2}\left[\cos \left(z \nabla_{j}\right) \nabla_{j}^{2} g_{3}^{j}\right] \mathbf{k}
\end{aligned}
$$

The displacement in the plate can be expressed as:

$$
\mathbf{u}=2(1-v)\left(\nabla_{1}^{2}+\frac{\partial^{2}}{\partial z^{2}}\right) \mathbf{G}-\nabla_{0}\left(\nabla_{0} \cdot \mathbf{G}\right) .
$$

Its component-wise expressions can be written as:

$$
\begin{aligned}
& u_{x}=\frac{\sin \left(z \nabla_{2}\right)}{\nabla_{2}} T_{2}^{2} g_{1}^{2}+\sum_{j=1}^{2} \frac{\sin \left(z \nabla_{j}\right)}{\nabla_{j}} \nabla_{j}^{2} \frac{\partial}{\partial x} g_{3^{\prime}} \\
& u_{y}=\frac{\sin \left(z \nabla_{2}\right)}{\nabla_{2}} T_{2}^{2} g_{2}^{2}+\sum_{j=1}^{2} \frac{\sin \left(z \nabla_{j}\right)}{\nabla_{j}} \nabla_{j}^{2} \frac{\partial}{\partial y} g_{3}^{j}
\end{aligned}
$$




$$
u_{z}=\cos \left(z \nabla_{2}\right) T_{2}^{2} g_{3}^{2}+\sum_{j=1}^{2} \cos \left(z \nabla_{j}\right) \nabla_{j}^{2} g_{3}^{j}
$$

Considering the neutral surface displacement and the normal angle, the generalized displacement in the plate can be expressed as:

$$
\begin{gathered}
\psi_{x}=-\left.\frac{\partial u_{x}}{\partial z}\right|_{z=0}=-T_{2}^{2} g_{1}^{2}-\sum_{j=1}^{2} \nabla_{j}^{2} \frac{\partial^{2} g_{3}^{j}}{\partial x}, \\
\psi_{y}=-\left.\frac{\partial u_{y}}{\partial z}\right|_{z=0}=-T_{2}^{2} g_{2}^{2}-\sum_{j=1}^{2} \nabla_{j}^{2} \frac{\partial^{2} g_{3}^{j}}{\partial y}, \\
w=\left.u_{z}\right|_{z=0}=\nabla^{2} g_{3}^{2}+\nabla_{1}^{2} g_{3}^{1} .
\end{gathered}
$$

The rotational normal angle to the neutral surface can be expressed as:

$$
\psi_{x}=\frac{\partial F}{\partial x}+\frac{\partial f}{\partial y}, \psi_{y}=\frac{\partial F}{\partial y}-\frac{\partial f}{\partial x} .
$$

The functions $g_{1}^{2}, g_{2}^{2}, g_{3}^{2}$ can be expressed by the neutral surface displacement and normal angle as:

$$
\begin{gathered}
g_{1}^{2}=-T_{2}^{-2}\left(\frac{\partial f}{\partial y}+\frac{\partial E}{\partial x}\right), \\
g_{2}^{2}=T_{2}^{-2}\left(\frac{\partial f}{\partial x}+\frac{\partial E}{\partial y}\right), \\
g_{3}^{1}=-T_{2}^{-2} \nabla_{1}^{-2}\left(\nabla_{1}^{2} w+\nabla^{2} F\right), \\
g_{3}^{2}=T_{2}^{-2}(F+w-E) .
\end{gathered}
$$

where $\nabla^{2} E=0, F=-\nabla_{1}^{2} g_{3}^{1}-\nabla_{2}^{2} g_{3}^{2}+E$.

In this way, the displacement can be derived as:

$$
\begin{aligned}
u_{x}= & \sum_{j=1}^{2}(-1)^{j-1} \frac{\sin \left(z \nabla_{j}\right)}{\nabla_{j}} \frac{\partial w}{\partial x}-\frac{\sin \left(z \nabla_{2}\right)}{\nabla_{2}}\left(\frac{\partial F}{\partial x}+\frac{\partial w}{\partial x}\right) \\
& -T_{2}^{-2} \sum_{j=1}^{2}(-1)^{j-1} \frac{\sin \left(z \nabla_{j}\right)}{\nabla_{j}} \nabla^{2}\left(\frac{\partial F}{\partial x}+\frac{\partial w}{\partial x}\right) \\
u_{y}= & \sum_{j=1}^{2}(-1)^{j-1} \frac{\sin \left(z \nabla_{j}\right)}{\nabla_{j}} \frac{\partial w}{\partial y}-\frac{\sin \left(z \nabla_{2}\right)}{\nabla_{2}}\left(\frac{\partial F}{\partial y}-\frac{\partial f}{\partial x}\right) \\
& -T_{2}^{-2} \sum_{j=1}^{2}(-1)^{j-1} \frac{\sin \left(z \nabla_{j}\right)}{\nabla_{j}} \nabla^{2}\left(\frac{\partial F}{\partial y}+\frac{\partial w}{\partial y}\right) \\
u_{z}= & \cos \left(z \nabla_{1}\right) w-T_{2}^{-2} \sum_{j=1}^{2}(-1)^{j-1} \cos \left(z \nabla_{2}\right) \nabla^{2}(F+w) .
\end{aligned}
$$

In line with Hooke's law, the stress components can be expressed as:

$$
\begin{aligned}
\tau_{z x}= & 2 \mu \cos \left(z \nabla_{1}\right) \frac{\partial w}{\partial x}-\mu \cos \left(z \nabla_{2}\right)\left(\frac{\partial F}{\partial x}+\frac{\partial w}{\partial x}+\frac{\partial f}{\partial y}\right) \\
& -2 \mu T_{2}^{-2} \sum_{j=1}^{2}(-1)^{j-1} \cos \left(z \nabla_{j}\right) \nabla^{2}\left(\frac{\partial F}{\partial x}+\frac{\partial w}{\partial x}\right)
\end{aligned}
$$




$$
\begin{gathered}
\tau_{z y}=2 \mu \cos \left(z \nabla_{1}\right) \frac{\partial w}{\partial y}-\mu \cos \left(z \nabla_{2}\right)\left(\frac{\partial F}{\partial y}+\frac{\partial w}{\partial y}-\frac{\partial f}{\partial x}\right) \\
-2 \mu T_{2}^{-2} \sum_{j=1}^{2}(-1)^{j-1} \cos \left(z \nabla_{j}\right) \nabla^{2}\left(\frac{\partial F}{\partial y}+\frac{\partial w}{\partial y}\right) \\
\sigma_{z}=2 \mu T_{2}^{-2} \sum_{j=1}^{2}(-1)^{j-1} \frac{\sin \left(z \nabla_{j}\right)}{\nabla_{j}} \nabla^{2} \nabla^{2}(F+w) \\
-(\lambda+2 \mu) T_{1}^{-2} T_{2}^{-2} \frac{\sin \left(z \nabla_{1}\right)}{\nabla_{1}} \nabla^{2}(F+w) \\
+(\lambda+2 \mu) T_{1}^{2} \frac{\sin \left(z \nabla_{1}\right)}{\nabla_{1}} w \\
-2 \mu \frac{\sin \left(z \nabla_{1}\right)}{\nabla_{1}} \nabla^{2} w+2 \mu \frac{\sin \left(z \nabla_{2}\right)}{\nabla_{2}} \nabla^{2}(F+w)
\end{gathered}
$$

Considering the free boundary condition, the shear stress at the plate surface is zero. The equalities can be given from Equations (32) and (33) as follows:

$$
\begin{aligned}
& {\left[\begin{array}{l}
\cos \left(\frac{h}{2} \nabla_{1}\right) \frac{\partial w}{\partial x}-\frac{1}{2} \cos \left(\frac{h}{2} \nabla_{2}\right)-T_{2}^{-2} \sum_{j=1}^{2}(-1)^{j-1} \cos \left(\frac{h}{2} \nabla_{j}\right) \nabla^{2} \\
\left(\frac{\partial F}{\partial x}+\frac{\partial w}{\partial x}\right)-\frac{1}{2} \cos \left(\frac{h}{2} \nabla_{2}\right) \frac{\partial f}{\partial y}=0
\end{array}\right.} \\
& {\left[\begin{array}{l}
\cos \left(\frac{h}{2} \nabla_{1}\right) \frac{\partial w}{\partial y}-\frac{1}{2} \cos \left(\frac{h}{2} \nabla_{2}\right)-T_{2}^{-2} \sum_{j=1}^{2}(-1)^{j-1} \cos \left(\frac{h}{2} \nabla_{j}\right) \nabla^{2} \\
\left(\frac{\partial F}{\partial y}+\frac{\partial w}{\partial y}\right)+\frac{1}{2} \cos \left(\frac{h}{2} \nabla_{2}\right) \frac{\partial f}{\partial x}=0
\end{array}\right.}
\end{aligned}
$$

where $h$ is the thickness of the plate.

On the basis of the complex variable function theory, Equations (35) and (36) can be regarded as the real part and imaginary part, which consist of a Riemann condition of the analytic function. Now, the non-homogeneous solution of the equation does not influence the solution of the stress state, so there can be:

$$
\begin{aligned}
& \cos \left(\frac{h}{2} \nabla_{1}\right) w-\left[\frac{1}{2} \cos \left(\frac{h}{2} \nabla_{2}\right)\right.\left.+T_{2}^{-2} \sum_{j=1}^{2}(-1)^{j-1} \cos \left(\frac{h}{z} \nabla_{j}\right) \nabla^{2}\right](F+w)=0, \\
& \cos \left(\frac{h}{2} \nabla_{2}\right) f=0 .
\end{aligned}
$$

According to the integral function theory, Equation (38) can be expanded in series as:

$$
\prod_{m=1}^{\infty}\left[1-\frac{h^{2} \nabla_{2}^{2}}{(2 m-1)^{2} \pi^{2}}\right] f=0 .
$$

By means of truncating the infinite series, the following second-order elastic wave equation is given as:

$$
\nabla^{2} f-\left(\frac{\pi^{2}}{h^{2}}+\frac{1}{c_{2}^{2}} \frac{\partial^{2}}{\partial t^{2}}\right) f=0 .
$$

According to the free boundary condition that the normal stress at the plate surface is zero, the governing equation for the elastic wave of the plate can be derived from Equations (34) and (37) as:

$$
\begin{aligned}
& T_{2}^{-2} \sum_{j=1}^{2}(-1)^{j-1} \cos \left(\frac{h}{2} \nabla_{j}\right) \nabla^{2}(F+w)+\frac{1}{2} \cos \left(\frac{h}{2} \nabla_{2}\right)(F+w)-\cos \left(\frac{h}{2} \nabla_{2}\right) w=0, \\
& T_{2}^{-2} \sum_{j=1}^{2}(-1)^{j-1} \frac{\sin \left(\frac{h}{2} \nabla_{j}\right)}{\nabla_{j}} \nabla^{2} \nabla^{2}(F+w)-\frac{1}{2}\left[\frac{\sin \left(\frac{h}{2} \nabla_{1}\right)}{\nabla_{1}}-\frac{2 \sin \left(\frac{h}{2} \nabla_{2}\right)}{\nabla_{2}}\right] \nabla^{2}(F+w) \\
& -\frac{\sin \left(\frac{h}{2} \nabla_{1}\right)}{\nabla_{1}} \nabla^{2} w+\frac{1}{2} T_{2}^{2} \frac{\sin \left(\frac{h}{2} \nabla_{1}\right)}{\nabla_{1}} w=0
\end{aligned}
$$


The operator algebraic equation involving the unknown functions $F$ and $w$ is deduced by Equations (41) and (42) as:

$$
\boldsymbol{\Lambda}\left[\begin{array}{c}
F \\
w
\end{array}\right]=\left[\begin{array}{l}
0 \\
0
\end{array}\right],
$$

where the expression of each operator of $\Lambda$ is

$$
\begin{aligned}
& \Lambda_{11}=T_{2}^{-2} \sum_{j=1}^{2}(-1)^{j-1} \cos \left(\frac{h}{2} \nabla_{j}\right) \nabla^{2}+\frac{1}{2} \cos \left(\frac{h}{2} \nabla_{2}\right), \\
& \Lambda_{12}=T_{2}^{-2} \sum_{j=1}^{2}(-1)^{j-1} \cos \left(\frac{h}{2} \nabla_{j}\right) \nabla^{2}-\cos \left(\frac{h}{2} \nabla_{1}\right)+\frac{1}{2} \cos \left(\frac{h}{2} \nabla_{2}\right), \\
& \Lambda_{21}=T_{2}^{-2} \sum_{j=1}^{2}(-1)^{j-1} \frac{\sin \left(\frac{h}{2} \nabla_{j}\right)}{\nabla_{j}} \nabla^{2} \nabla^{2}-\frac{1}{2} \frac{\sin \left(\frac{h}{2} \nabla_{1}\right)}{\nabla_{1}} \nabla^{2}+\frac{\sin \left(\frac{h}{2} \nabla_{2}\right)}{\nabla_{2}} \nabla^{2}, \\
& \Lambda_{22}=T_{2}^{-2} \sum_{j=1}^{2}(-1)^{j-1} \frac{\sin \left(\frac{h}{2} \nabla_{j}\right)}{\nabla_{j}} \nabla^{2} \nabla^{2}-\frac{3}{2} \frac{\sin \left(\frac{h}{2} \nabla_{1}\right)}{\nabla_{1}} \nabla^{2}+\frac{\sin \left(\frac{h}{2} \nabla_{2}\right)}{\nabla_{2}} \nabla^{2}+\frac{1}{2} T_{2}^{2} \frac{\sin \left(\frac{h}{2} \nabla_{1}\right)}{\nabla_{1}} \\
& \text { The determinant of Equation (23) is: }
\end{aligned}
$$

$$
\begin{aligned}
\operatorname{det}(\boldsymbol{\Lambda}) & =T_{2}^{-2}\left[\frac{\sin \left(\frac{h}{2} \nabla_{1}\right)}{\nabla_{1}} \cos \left(\frac{h}{2} \nabla_{2}\right)-\frac{\sin \left(\frac{h}{2} \nabla_{2}\right)}{\nabla_{2}} \cos \left(\frac{h}{2} \nabla_{1}\right)\right] \nabla^{2} \nabla^{2} \\
& -\left[\frac{\sin \left(\frac{h}{2} \nabla_{1}\right)}{\nabla_{1}} \cos \left(\frac{h}{2} \nabla_{2}\right)-\frac{\sin \left(\frac{h}{2} \nabla_{2}\right)}{\nabla_{2}} \cos \left(\frac{h}{2} \nabla_{1}\right)\right] \nabla^{2} \\
& +\frac{1}{4} T_{2}^{2} \frac{\sin \left(\frac{h}{2} \nabla_{1}\right)}{\nabla_{1}} \cos \left(\frac{h}{2} \nabla_{2}\right)
\end{aligned}
$$

The fourth-order differential equation involving the lateral displacement can be expressed as:

$$
\operatorname{det}(\boldsymbol{\Lambda}) w=0
$$

After the truncation of the infinite order operator series, the governing equation for the elastic wave of plates can be derived as:

$$
\begin{gathered}
\nabla^{2} \nabla^{2} w-\frac{3-2 \kappa}{2(1-\kappa)} T_{2}^{2} \nabla^{2} w+\frac{3}{1-\kappa} T_{2}^{2}\left(\frac{1}{h^{2}}+\frac{1}{24} T_{1}^{2}+\frac{1}{8} T_{2}^{2}\right) w=0 \\
C \nabla^{2} \nabla^{2} w-(2-v) D T_{2}^{2} \nabla^{2} w+\left[C T_{2}^{2}+\left(\frac{7}{8}-v\right) D T_{2}^{4}\right] w=0
\end{gathered}
$$

where $C=\frac{E_{\mathrm{M}} h}{2(1+v)}, D=\frac{E_{\mathrm{M}} h}{12\left(1-v^{2}\right)}, E_{\mathrm{M}}$ is Young modulus.

Without loss of generality, the solution of the vibration harmonic of the problem is studied. Set:

$$
w=\widetilde{w} \mathrm{e}^{-\mathrm{i} \omega t}, \quad F=\widetilde{F} \mathrm{e}^{-\mathrm{i} \omega t}, f=\widetilde{f} \mathrm{e}^{-\mathrm{i} \omega t},
$$

where $\omega$ is the angular frequency of plate bending, and i is an imaginary unit.

In the following analysis, the time factor and the symbol ' $\sim$ ' in the generalized displacement functions are left out. Taking Equation (48) into Equation (47), the following equations can be expressed as:

$$
\begin{gathered}
\nabla^{2} \nabla^{2} w+\frac{3-2 \kappa}{2(1-\kappa)} k_{2}^{2} \nabla^{2} w+\frac{3}{4(1-\kappa)} k_{2}^{2}\left(\frac{\kappa k_{2}^{2}}{6}+\frac{k_{2}^{2}}{2}-\frac{4}{h^{2}}\right) w=0 \\
\prod_{j=1}^{2}\left(\nabla^{2}+\alpha_{j}^{2}\right) w=0
\end{gathered}
$$

where $\alpha_{j}(j=1,2)$ are scattering wave numbers, which satisfy the following expression $\alpha^{4}-(2-v) k_{2}^{2} \alpha^{2}+k_{2}^{2}\left[\frac{(7-8 v) k_{2}^{2}}{8}-\frac{6(1-v)}{h^{2}}\right]=0$. 
The scattering numbers on the basis of Mindlin plate theory are determined by the following expression: $\alpha^{4}-\left(\frac{12}{\pi^{2}}+\frac{1-v}{2}\right) k_{2}^{2} \alpha^{2}+6(1-v) k_{2}^{2}\left(\frac{k_{2}^{2}}{\pi^{2}}-\frac{1}{h^{2}}\right)=0$, where $k_{j}=\frac{\omega}{c_{j}}$, $(j=1,2)$.

The corresponding generalized displacement potential function is:

$$
F=F_{1}+F_{2}=\sum_{j=1}^{2}\left(\delta_{j}-1\right) w_{j}
$$

where $\delta_{j}$ are the ratio coefficients of the displacement potential function, and thus $\delta_{j}=\frac{16+2\left(\alpha_{j}^{2}-\kappa k_{2}^{2}\right) h^{2}}{8+\left[(3-2 \kappa) \alpha_{j}^{2}-k_{2}^{2}\right] h^{2}}$.

Comparatively, the ratio coefficients of the displacement potential function by Mindlin plate theory are $\delta_{1}=-\frac{2}{1-v} \frac{\alpha_{2}^{2} h^{2}}{\pi^{2}-k_{2}^{2} h^{2}}, \delta_{2}=-\frac{2}{1-v} \frac{\alpha_{1}^{2} h^{2}}{\pi^{2}-k_{2}^{2} h^{2}}$.

\section{Comparison of Various Bending Plate Theories}

In this paper, the comparisons between the governing equation for the bending plate and the governing equations for various classical bending plates are presented in Table 1.

Table 1. Comparison of different theories of plate bending.

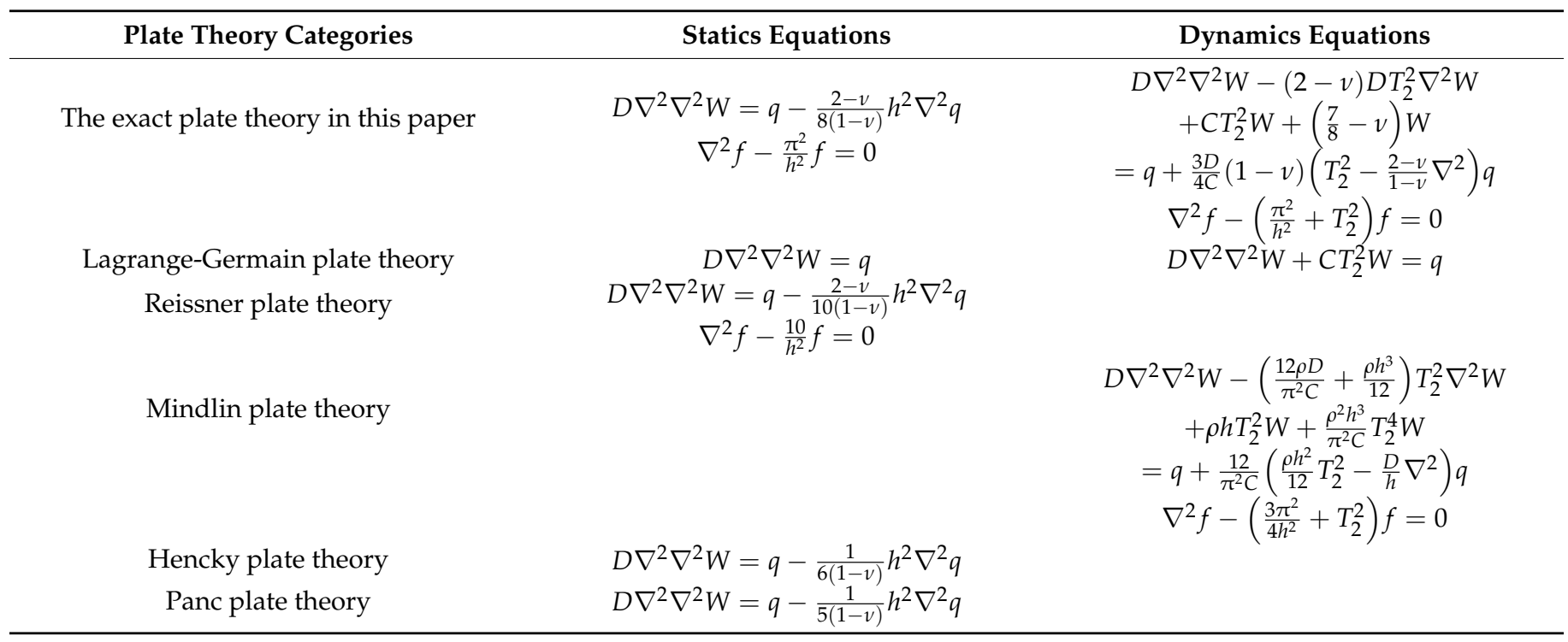

The dispersion equation based on the Lagrange-Germain plate theory (CPT) can be described as:

$$
\frac{c}{c_{2}}=\sqrt{\frac{4 \pi^{2}}{6(1-v)} \frac{h}{\lambda}} .
$$

where $\lambda$ is the wavenumber of an elastic wave.

The dispersion equation in consideration of the moment of inertia can be described as:

$$
\frac{c}{c_{2}}=\sqrt{\frac{4 \pi^{2}}{6(1-v)}\left[1+\frac{\pi^{2}}{3}\left(\frac{h}{\lambda}\right)^{2}\right]^{-1}} \frac{h}{\lambda} .
$$


When the moment of inertia and shear deformation are involved, the implicit dispersion equation can be described as:

$$
\frac{\pi^{2}}{3}\left(\frac{h}{\lambda}\right)^{2}\left(1-\frac{c^{2}}{\kappa^{2} c_{2}^{2}}\right)\left(\frac{c_{p}^{2}}{c^{2}}-1\right)=1 .
$$

The implicit dispersion equation based on the three-dimensional elasto-dynamics theory can be described as:

$$
\frac{4 c_{2}^{2} \sqrt{\left(c_{2}^{2}-\kappa c^{2}\right)\left(c_{2}^{2}-c^{2}\right)}}{\left(2 c_{2}^{2}-c^{2}\right)^{2}}=\frac{\tanh \left(2 \pi \frac{h}{\lambda} \sqrt{c_{2}^{2}-\kappa c^{2}}\right)}{\tanh \left(2 \pi \frac{h}{\lambda} \sqrt{c_{2}^{2}-c^{2}}\right)} .
$$

The dispersion equation based on the exact plate theory in this paper is:

$$
\alpha^{4}-(2-v) k_{2}^{2} \alpha^{2}+(1-v) k_{2}^{2}\left[\frac{k_{2}^{2}(7-8 v)}{8(1-v)}-\frac{6}{h^{2}}\right]=0 .
$$

\section{Discussion of the Exact Plate Theory}

In this paper, the derived plate bending vibration equation is compared with the classical corresponding equation. The comparison of the specific equation form is shown in Table 1. In the process of comparison, the equation form in the frequency domain is used. The bending vibration equation of plates presented in this paper is similar to other classical bending vibration equations of plates. When the statics problem is studied, the elastic vibration equation of plates derived in this paper degenerates into an exact equation of the static bending of plates.

According to those dispersion equations above, Figure 1 is drawn to compare the dispersion curves; Figure 2 is drawn to compare the scattering wave numbers. From Figure 1, we can see that the dispersion curves based on the classic thin plate theory and Mindlin plate theory are far apart from the three-dimensional elasto-dynamics theory, but the dispersion curves by the exact plate theory in this paper are very close to the dispersion curves based on the three-dimensional elasto-dynamics theory. By comparing those curves, the superiority of the exact plate theory to other plate theories is obvious.

As can be seen in Figure 2, the scattering wave number $\alpha_{1}$ obtained by the exact plate theory and Mindlin plate theory is very close, but the scattering wave number $\alpha_{2}$ obtained by the exact plate theory is quite different from that of the Mindlin plate theory. With the increase of the vibration frequency, the scattering wave number becomes greater. It can be seen that the scattering wave number $\alpha_{1}$ at any frequency is greater than zero, so it can be concluded that the wave mode is in the propagation region. Nevertheless, when the frequency is low, the scattering wave number $\alpha_{2}$ is less than zero, and the wave mode is in the cutoff frequency domain, which is called a localized standing wave. When the frequency is high, the scattering wave number $\alpha_{2}$ is greater than zero, and the wave mode is in the propagation region, which is called a propagating wave. According to Reference [7], it can be seen that the applicable frequency interval of the Mindlin plate theory is $\omega / \omega_{0}<1$, i.e., $h / \lambda_{2}<0.5$, and the application of the Mindlin plate theory is limited. The dynamic model proposed in this paper is completely based on the three-dimensional elasto-dynamics theory, and consequently, its limitations of application are minor. 


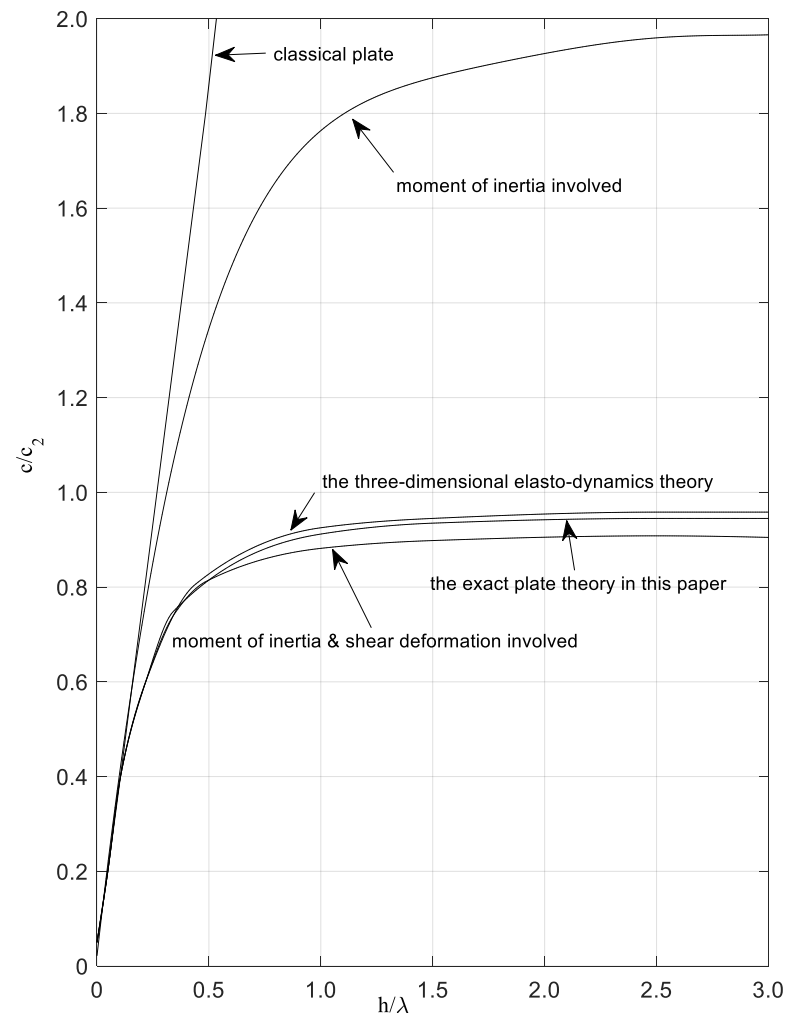

Figure 1. Dispersion relation by the different theories.

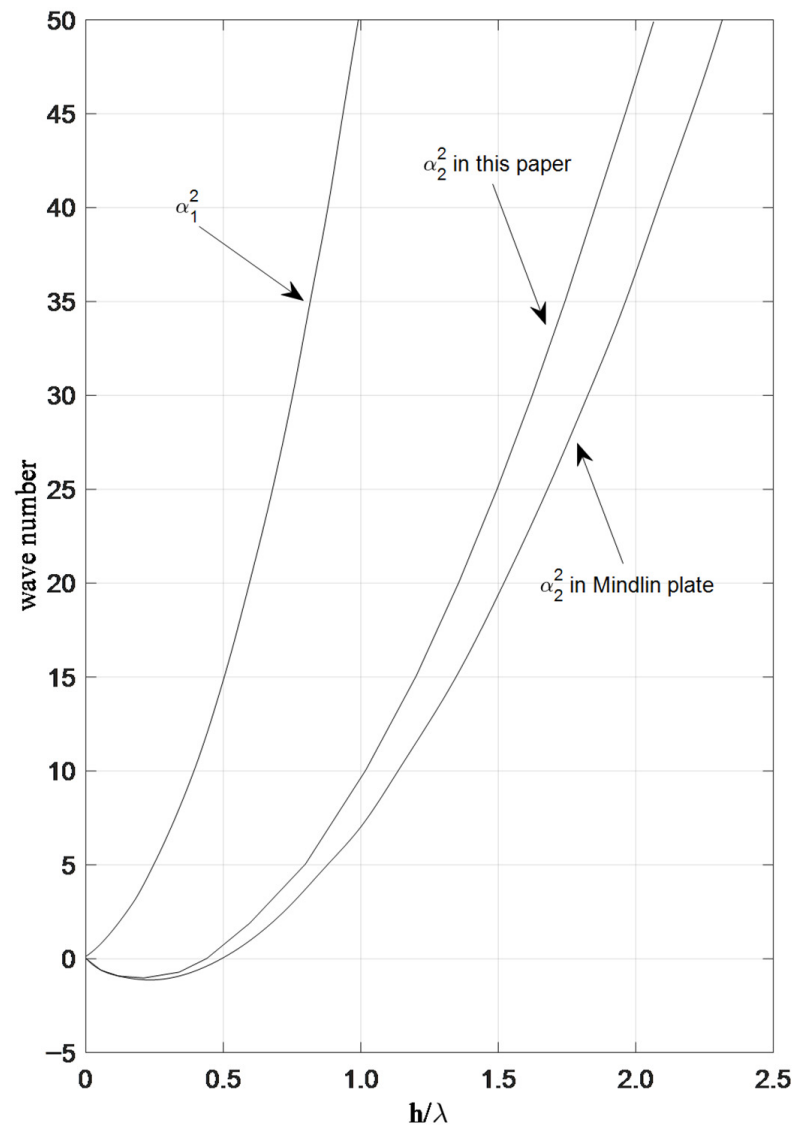

Figure 2. Wavenumbers by the different theories. 


\section{Conclusions}

In this paper, the novel dynamics plate model is completely based on the theory of three-dimensional elasto-dynamics theory, and the derivation of the dynamic equation is conducted without any prior assumption but in view of the partial differential operator theory and analytic theory of complex function. Therefore, the proposed dynamic equation is more exact. It is not only suitable for low-frequency vibration of the plate but also suitable for high-frequency vibration, which will not produce the phenomenon of high-frequency dispersion.

With the development of modern mechanics and mathematics, new research results and methods continue to appear. The research object develops toward high speed and high-frequency. The new development of mathematical and mechanics improves the ability to solve complex mechanical problems and plays a positive role in promoting the development of mathematics in mechanics and physics. It provides a new formulation and means for solving the dynamics and numerical calculation of solid structures more accurately. The governing equation of bending plate vibration proposed in this paper is expected to be used to analyze the vibration of thick plates, evaluate the applicable condition of the engineering plate theory and design the active vibration control of thick plates exactly in the research on the dynamics and stability for flexible spacecraft structures and broadband vibration frequency.

Author Contributions: Conceptualization, C.Z. and M.W.; validation, C.Z. and M.W.; investigation, X.H. and W.Z.; data curation, X.H. and H.X.; writing—original draft preparation, C.Z.; writingreview and editing, M.W. and J.N.; funding acquisition, J.N. All authors have read and agreed to the published version of the manuscript.

Funding: This research was supported by the Key Laboratory of Underwater Acoustic Environment Institute of Acoustic Chinese Academy of Science SSHJ-KFKT-2020, the Key Research and Development Program of Zhejiang Province (No. 2021C03013), the National Key Research \& Development Program of China (No. 2017YFB1301300), the Key Laboratory funding for Technology in Rural Water Management of Zhejiang Province and the Fundamental Research Funds for the Provincial Universities of Zhejiang (GK199900299012-026).

Institutional Review Board Statement: The study in this paper did not involve humans or animals.

Informed Consent Statement: Not applicable.

Data Availability Statement: The study did not report any data.

Conflicts of Interest: The authors declare no conflict of interest.

\section{References}

1. Corral, E.; García, M.J.G.; Castejon, C.; Meneses, J.; Gismeros, R. Dynamic modeling of the dissipative contact and friction forces of a passive biped-walking robot. J. Appl. Sci. 2020, 10, 2342. [CrossRef]

2. Corral, E.; Moreno, R.G.; García, M.J.G.; Castejón, C. Nonlinear phenomena of contact in multibody systems dynamics: A review. Nonlinear Dyn. 2021, 104, 1269-1295. [CrossRef]

3. Kolahchi, R. A comparative study on the bending, vibration and buckling of viscoelastic sandwich nano-plates based on dif-ferent nonlocal theories using DC, HDQ and DQ methods-ScienceDirect. J. Aerosp. Sci. Technol. 2017, 66, 235-248. [CrossRef]

4. Nicassio, F. Shape prediction of bistable plates based on Timoshenko and Ashwell theories. Compos. Struct. 2021, $265,113645$. [CrossRef]

5. Wu, C.; Viquerat, A.; Aglietti, G. Natural Frequency Optimization and Stability Analysis of Bistable Carbon Fiber Reinforced Plastic Booms for Space Applications. In Proceedings of the 3rd AIAA Spacecraft Structures Conference, San Diego, CA, USA, 4-8 January 2016. [CrossRef]

6. Saada, A.S. Elasticity: Theory and Applications; J. Ross Pub.: Ft. Lauderdale, FL, USA, 2009.

7. Pao, Y.H.; Mow, C.C. Diffraction of elastic wave and dynamic stress concentration. J. Appl. Math. 1973, 872. [CrossRef]

8. Shimpi, R.P.; Shetty, R.A.; Guha, A. A single variable refined theory for free vibrations of a plate using inertia related terms in displacements. Eur. J. Mech. A-Solid 2017, 65, 136-148. [CrossRef]

9. Wang, C.M.; Kitipornchai, S. Frequency relationship between Levinson plates and classical thin plates. Mech. Res. Commun. 1999, 26, 687-692. [CrossRef] 
10. Wang, C.M.; Reddy, J.N.; Lee, K.H. (Eds.) Shear Deformable Beams and Plates: Relationships with Classical Solutions, 1st ed.; Elsevier: Amsterdam, The Netherlands, 2000.

11. Reissner, E. On the Theory of Bending of Elastic Plates. J. Math. Phys. 1944, 23, 184-191. [CrossRef]

12. Reissner, E. The Effect of Transverse Shear Deformation on the Bending of Elastic Plates. J. Appl. Mech. 1945, 12, A69-A77. [CrossRef]

13. Mindlin, R.D. Influence of rotary inertia and shear on flexural motions of isotropic elastic plates. J. Appl. Mech. 1951, 18, 31-38. [CrossRef]

14. Reissner, E. Reflections on the Theory of Elastic Plates. Appl. Mech. Rev. 1985, 38, 1453-1464. [CrossRef]

15. Levinson, M. An accurate, simple theory of the statics and dynamics of elastic plates. Mech. Res. Commun. 1980, 7, 343-350. [CrossRef]

16. Reddy, J.N. A Simple Higher-Order Theory for Laminated Composite Plates. J. Appl. Mech. 1984, 51, 745-752. [CrossRef]

17. Reddy, J. A refined nonlinear theory of plates with transverse shear deformation. Int. J. Solids Struct. 1984, 20, 881-896. [CrossRef]

18. Kant, T. Numerical analysis of thick plates. Comput. Methods Appl. Mech. Eng. 1982, 31, 1-18. [CrossRef]

19. Wang, X.; Shi, G. A refined laminated plate theory accounting for the third-order shear deformation and interlaminar transverse stress continuity. Appl. Math. Model. 2015, 39, 5659-5680. [CrossRef]

20. Nedri, K.; Meiche, N.; Tounsi, A. Free vibration analysis of laminated composite plates resting on elastic foundations by using a refined hyperbolic shear deformation theory. J. Mech Compos Mater. 2014, 49, 629-640. [CrossRef]

21. Shimpi, R.P. Refined plate theory and its variants. AIAA J. 2002, 40, 137-146. [CrossRef]

22. Shimpi, R.P.; Patel, H.G. Free vibrations of plate using two variable refined plate theory. J. Sound Vibr. 2006, 296, 979-999. [CrossRef]

23. Carrera, E.; Filippi, M.; Zappino, E. Free vibration analysis of rotating composite blades via Carrera Unified Formulation. Compos. Struct. 2013, 106, 317-325. [CrossRef]

24. Tornabene, F.; Viola, E.; Fantuzzi, N. General higher-order equivalent single layer theory for free vibrations of doubly-curved laminated composite shells and panels. Compos. Struct. 2013, 104, 94-117. [CrossRef]

25. Tornabene, F.; Fantuzzi, N.; Viola, E.; Carrera, E. Static analysis of doubly-curved anisotropic shells and panels using CUF approach, differential geometry and differential quadrature method. Compos. Struct. 2014, 107, 675-697. [CrossRef] 